\title{
Neck/shoulder function in tension-type headache patients and the effect of strength training
}

This article was published in the following Dove Press journal: Journal of Pain Research

\section{Bjarne K Madsen' \\ Karen Søgaard 2,3 \\ Lars L Andersen ${ }^{4}$ \\ Jørgen Skotte ${ }^{4}$ \\ Birte Tornøe ${ }^{5}$ \\ Rigmor $\mathrm{H}$ Jensen'}

'Danish Headache Center, Department of Neurology, University of Copenhagen, RigshospitaletGlostrup, Glostrup, ${ }^{2}$ Institute of Sports Science and Clinical Biomechanics, Physical Activity and Health in Work Life University of Southern Denmark, Odense, ${ }^{3}$ Department of Clinical research, University of Southern Denmark, Odense, ${ }^{4}$ National Research Centre for the Working Environment, Copenhagen, Denmark; ${ }^{5}$ Department of Health Sciences, Lund University, Lund, Sweden
Correspondence: Bjarne Kjeldgaard Madsen

Danish Headache Center, Department of Neurology, Rigshospitalet Glostrup, Nordre Ringvej 69, 2600 Glostrup, Denmark

Email bjarne.kjeldgaard.madsen@regionh. $\mathrm{dk}$
Introduction: Muscle pain has been associated with reduced maximal muscle strength, and reduced rate of force development (RFD). Strength training (ST) has shown an effect in not only normalizing muscle function but also reducing neck muscle pain.

Aim: The aims of this study were to compare muscle function in terms of strength, force steadiness in neck flexion, as well as extension, and rate of RFD of the shoulder in tension-type headache (TTH) patients and healthy controls and to examine the correlation to tenderness. Furthermore, the aim of the study was to examine the effect of ST on neck and shoulder functions in TTH patients.

Participants and methods: In all, 60 TTH patients and 30 sex- and age-matched healthy controls were included for a case-control comparison. The 60 patients with TTH were randomized into an ST and an ergonomic and posture correction (EP) control group. The ST group trained for 10 weeks with elastic bands.

Results: TTH patients had a lower extension force steadiness with a significant $15 \%$ higher coefficient of variation $(\mathrm{CoV})$ compared to healthy controls $(p=0.047)$. A significantly lower RFD (25\%) was noted in the TTH group than in the healthy controls $(p=0.031)$. A significant $(p<0.01)$ and moderate correlation to muscle tenderness was found. In the intervention, 23 patients completed ST and 21 patients completed EP. No significant between-group effect was observed, but at 22 weeks follow-up, both groups had a significant within-group effect of improved extension force steadiness (ST: $p=0.011$ and EP: $p<0.01$ ).

Conclusion: TTH patients showed a deteriorated muscle function, indicated by a lower force steadiness and RFD, compared to the healthy controls. The effect of ST was not larger than EP as both groups of TTH patients showed some improvement in neck and shoulder functions during the 10 weeks intervention and at follow-up. Future interventions are needed to elucidate if normalization of muscle function can lead to a reduction in headache.

Keywords: tension-type headache, force steadiness, rate of force development, strength training, ergonomic, posture correction

\section{Introduction}

Tension-type headache (TTH) is very common and has a large impact on the everyday life of the general population, with a substantial influence on work, leisure and social activities. ${ }^{1-4}$ The muscles are considered to be one of the most important factors to influence TTH. ${ }^{5,6}$ The muscle tenderness is considered to have an important influence on TTH, with an increasing tenderness with increasing headache intensity and frequency. ${ }^{7,8}$ A population-based study confirmed that patients with chronic TTH (CTTH) have a significantly higher total tenderness score (TTS) compared to the general population. ${ }^{31}$ 
A high prevalence of headache has been found in individuals with musculoskeletal symptoms in the neck in a Norwegian study, and this has been confirmed in a Danish study. ${ }^{9,10}$

The ability to control and sustain activation of adequate muscle force depends highly on the proprioceptive system that may be impaired in TTH patients. ${ }^{11}$ The ability to sustain a muscle contraction with minimum force fluctuations while matching a given force level can be measured as the coefficient of variation $(\mathrm{CoV})$ of the force over time and is referred to as force steadiness. It has been demonstrated that females with neck pain have a reduced force steadiness that may be caused by alterations in the afferent input from neck joint or muscle receptors. ${ }^{11}$

An increased co-activation of neck muscles has previously been found in females with CTTH compared to healthy individuals. ${ }^{12}$ This could indicate a changed motor control strategy and potentially a risk of muscle overload. ${ }^{12}$ In a previous study, we have described that the ratio of extension and flexion muscles was reduced in TTH patients compared to healthy controls. ${ }^{13}$ This decreased ratio may suggest a dysfunctional muscle activity, leading to a reduced force steadiness. Another indicator of neuromuscular control is the ability to recruit available muscle force as quickly as possible, which is referred to as rate of force development (RFD). Muscle pain in the neck region has been shown to influence the force-generating capacity. ${ }^{14,15}$ Unspecific pain in the neck region is often classified as trapezius myalgia, and patients with trapezius myalgia have shown a significantly reduced RFD over the shoulder joint. ${ }^{14,15}$ In a randomized controlled trial (RCT), a 10-week strength training (ST) program targeting muscle pain in patients with trapezius myalgia effectively reduced neck pain and increased muscle strength. ${ }^{16}$ Secondary analysis on similar training programs in headache patients with frequent episodic tension-type headache has showed a reduction in headache. ${ }^{23,32}$ The reduction in muscle pain through ST can be influenced by protein synthesis and degradation, which can lead to reconstruction of damaged muscle fiber. ${ }^{17,19}$ Furthermore, the increased strength may reduce the relative workload on the muscle in daily life. ${ }^{18}$ No studies have evaluated force steadiness and RFD as indicators of a deteriorated neuromuscular control in TTH patients, in spite of neck pain being a very common complaint in this patient group. ${ }^{10}$

\section{Aim \\ Case-control}

The aim of the present study was to compare TTH patients with healthy controls regarding force steadiness in neck flexion and extension as well as RFD of the shoulder.
More specifically, it was hypothesized that the TTH patients compared to healthy controls would have a reduced strength, force steadiness and RFD and that these indicators of deteriorated neuromuscular control would correlate with the level of total tenderness.

\section{RCT}

The aim was to evaluate the effect of ST in the TTH patients in a controlled design. It was hypothesized that the ST intervention would improve strength, force steadiness and RFD.

\section{Participants and methods Participants}

The study is a subanalysis of a study from 2010 to 2012 including a case-control and an RCT that was performed at the Danish Headache Center, Department of Neurology, Rigshospitalet-Glostrup. The participants received written and verbal information about the study, and all of them gave written informed consent. Ethics approval for the study was granted by the ethics committee of the Capital Region of Denmark: H-3-2009-080. This study is registered under clinical trials registration number NCT02984826.

The TTH patients were classified according to the International Classification of Headache Disorders, 2nd Edition (ICHD -II) criteria. ${ }^{20}$ It was aimed to include $60 \mathrm{TTH}$ patients and 30 healthy controls based on power calculations for the primary outcome in the intervention part of the study (frequency and duration presented previously). ${ }^{13,22}$ After the baseline case-control testing, the healthy controls exited the trial. In the RCT, the TTH patients were randomized by a random sequence generator into two groups, and the patients were not blinded to allocation. This paper presents results from analysis of secondary outcomes from the RCT study. Additionally, a case-control comparison of the baseline values of these secondary outcomes in TTH patients with a control group is included. Previously, similar analysis on the primary outcomes, headache frequency and duration has been published. ${ }^{13,22}$ Further medication intake was registered as a secondary outcome, and it has been analyzed and published that no significant reduction after intervention was found..$^{22}$ In addition, it was shown that there were patients with coexisting migraine and and these showed a similar decrease in force as those with only $\mathrm{TTH}^{13}$

The inclusion criteria were as follows:

1. age between 18 and 65 years;

2. TTH according to ICHD-II criteria (TTH $\geq 8$ headache days/month and $\leq 3$ migraine days/month). ${ }^{20}$ 
The exclusion criteria were as follows:

1. headache caused by medication overuse (ICHD-II);

2. previous whiplash or head trauma;

3. other major physical or neurological diseases, depression, and/or other psychiatric disorders;

4. inability to understand and speak Danish;

5. positive test for cervicogenic headache according to Jull et al. ${ }^{21}$

\section{Healthy controls}

The exclusion criteria were physical, neurological or psychiatric disorders and headaches $>1$ day/month.

The recruiting process has been described in detail previously. ${ }^{13,22}$

\section{Measurement methods}

A physiotherapist (BKM) included the participants and was blinded to the test results, whereas the tester (Medical Laboratory Technologist) was blinded to headache status. Both healthy controls and TTH patients were tested in a baseline screening involving neck, shoulder, total tenderness and joint mobility tests. The tests were conducted by the same tester in a standardized test battery.

The patients were subsequently tested 10 weeks and 22 weeks from baseline in the follow-up, with the same standardized test battery as at baseline, by the same tester.

Patients were not tested if presenting with migraine on the test day, but TTH was not a limitation.

All maximal voluntary contraction (MVC) tests were performed using dynamometer equipment connected to a computer that registered and stored all test data; this method has previously been described in detail. ${ }^{13}$

\section{Total tenderness score}

Palpation procedure of tenderness

The subjects were sitting in a chair with supported head, and the arms hanging freely. The palpation was performed with small rotation of the second and third fingers. The system has been described by Bendtsen. ${ }^{24}$ Tenderness scoring was established by palpating seven bilateral sites (the masseter, frontalis, temporalis, trapezius and sternocleidomastoid muscles; the processus mastoideus and the occipitalis muscles insertion) using a four point scale $(0-3)$ with a maximum of $7 \times 2 \times 3=42$ points.

\section{Neck test}

Neck force in extension and flexion was measured with the participant seated in an upright position on a chair with the chest strapped to a vertical plate and arms hanging relaxed in front of a custom-designed steel frame with an attachment arm and a strain guage force transducer. This is further described in a previous paper. ${ }^{13}$

\section{Force steadiness}

The maximal force in flexion and extension was used to establish a $30 \%$ target force line shown on a screen. The patients aimed to maintain the $30 \%$ MVC target force over 30 seconds, as stable as possible, guided by a visual feedback. The actual mean force and standard deviation (SD) were registered and used to calculate coefficient of variation as $\mathrm{SD} /$ mean over a 25 -second period. Three attempts were made. The result with the lowest $\mathrm{CoV}$ was chosen for each participant.

\section{RFD}

The MVC and the RFD of the shoulder were measured with the same equipment as described earlier. The testing was conducted with the participant lying supine. The measurement is further described in Madsen et al. ${ }^{13}$ For each trial, the RFD $\left(\mathrm{Nm} \times \mathrm{s}^{-1}\right)$ was determined as the steepest slope over $100 \mathrm{~ms}$ of the rising part of the filtered force-time curve. For statistical analysis, the trial with the highest RFD was chosen.

\section{Joint examination}

For joint examination, the validated Beighton score was used to measure the general joint mobility. It is conducted as a bilateral examination in a standing position of five joint movements that include the fifth metacarpophalangeal joint, thumb, elbow, knee and hands flat on the floor. The maximum score is 9 points. A positive finding of hypermobility was $\geq 5 / 9{ }^{25}$

\section{Intervention}

In this paper, only the secondary outcome measures of the intervention were included. Primary outcome was change in the headache frequency and duration obtained from a headache diary. This has, together with more details, been presented in a previous paper. ${ }^{22}$ Headache frequency and duration was at baseline recorded for the previous 4 weeks and compared to weeks 19-22 at follow-up (10 weeks intervention +12 weeks follow-up period). ${ }^{22}$ In short, the TTH patients were randomized by the random sequence generator into two groups: an ST group who conducted progressive ST for 10 weeks with elastic bands (TheraBand) and a control group who were instructed in ergonomic and posture corrections (EPs).

The specific ST group was trained and filled out a 10 -week training diary. The training consisted of ST three times per week. Days of training during the week were by own choice and were supervised weekly by a physiotherapist 
for the first 2 weeks and thereafter every second week. The ST consisted of four shoulder exercises. During the training period, the relative training load was progressively increased from 12 repetitions maximum (RMs; 70\% of maximal intensity) at the beginning to eight RMs ( $80 \%$ of the maximal intensity). ST was performed with slow concentric and eccentric muscle contractions with resistance from the elastic bands. The appropriate elastic band was selected and over time shortened or changed to meet the requirements of resistance. The elastic bands were handed in after the 10 weeks of training. The exercises have been described previously in more detail with pictures of the exercises. ${ }^{22}$

The EP control group was instructed in EP. They were instructed to pay attention to their sitting positions and how they used a computer or other working tools. Furthermore, they were asked to do a posture correction exercise three times a day with 10 repetitions. The purpose of the exercise was to increase consciousness of sitting positions. The exercise started with the patient in a posture with a flat lumbar lordosis with protruded shoulders and neck. Thereafter, they overcorrected the lumbar lordosis, shoulders and neck, and after 4 seconds slowly moved back to the starting posture. The control group was seen twice during the 10 -week period and was called over the phone every 2 weeks to ask if they paid attention to their ergonomics and posture correction, and filled out the headache diary. After 10 weeks, they were instructed that they should stop the exercise but continue to be conscious of their ergonomics and sitting position.

The ST intervention compared to EP resulted in no effect on the primary outcome of change in the headache frequency and duration as described previously. ${ }^{22}$

\section{Statistics}

Stata (StataCorp LP, College Station, TX, USA) was used to calculate statistics.

\section{Case control}

When data were not normally distributed, they were transformed, and a Unpaired $t$-test was used to test for significant difference between healthy controls and TTH patients. $p<0.05$ was used as the level of significance. It was transformed by using the Stata ladder function to fulfill the normality assumptions. In the text, data are presented as mean and SD values unless otherwise described. Regression analysis was used to determine if there was a significant association between total tenderness and measurements, and correlation was examined.

\section{RCT}

When data were not normally distributed, they were transformed by using the Stata ladder function to fulfill the normality assumptions. Repeated measures ANOVA was conducted, analyzing both between-subject and withinsubject effects. Regarding missing values, carry forward imputation was used.

\section{Results}

\section{Case-control study}

In all, 60 TTH patients were included; mean (SD) headache frequency was 18.6 (7.7) days/28 days and headache duration was 219 (142) hours/28 days. Among the 60 TTH patients, 25 had frequent episodic TTH (FETTH) and 35 had CTTH. A total of 30 healthy controls were included, and their demographics are presented in Table 1.

The subject recruitment process is presented in detail in a previous paper. ${ }^{13}$ All measurements of TTH patients and healthy controls are presented in Table 2 .

The test results show that the ability of fast force generation over the shoulder joint was affected in the TTH group with a significantly reduced RFD in the headache group compared to healthy controls (Table 2). Force steadiness was significantly affected in the extension with a higher $\mathrm{CoV}$ in the extension but not in flexion. The higher CoV result shows that the TTH patients were unable to maintain a steady force over 25 seconds. Total tenderness was as in other studies significantly higher than that in the healthy controls.

A highly significant association between total tenderness and the muscle function was found (Table 2). Scatterplots for

Table I Age and anthropometric measures of included TTH and healthy control subjects

\begin{tabular}{lllll}
\hline & TTH $(\mathbf{n = 6 0})$ & Healthy control $(\mathbf{n}=\mathbf{3 0})$ & ST $(\mathbf{n}=\mathbf{2 3})$ & EP $(\mathbf{n}=\mathbf{2 1})$ \\
\hline Females/males & $41 / 19$ & $21 / 9$ & $18 / 5$ & $15 / 6$ \\
Age (years) & $33.6 \pm 11.6$ & $35.6 \pm 14.7$ & $33 \pm 11.5$ & $36 \pm 10.8$ \\
Height $(\mathrm{cm})$ & $173 \pm 7.6$ & $175 \pm 9.1$ & $171 \pm 7$ & $174 \pm 7$ \\
Weight $(\mathrm{kg})$ & $72 \pm 16.4$ & $76 \pm 19.4$ & $71 \pm 14$ & $74 \pm 16$ \\
BMI $\left(\mathrm{kg} / \mathrm{m}^{2}\right)$, mean (SD) & $23.9 \pm 4.2$ & $24.5 \pm 4.54$ & $24 \pm 4$ & $24 \pm 5$ \\
\hline
\end{tabular}

Note: For TTH, data are also given separately for ST and EP.

Abbreviations: TTH, tension-type headache; ST, strength training; EP, ergonomic and posture correction; BMI, body mass index; SD, standard deviation. 
Table 2 Case-control measurements: TTH and healthy controls ( $p$ value between groups) and correlation between TTS and all measurements ( $p$ value for significant association, coefficient $[r]$ )

\begin{tabular}{llll}
\hline & & TTH & Healthy controls \\
\hline Measurements & TTS & $17.77 \pm 7.3$ & $4.73 \pm 5.02^{* *}$ \\
& Force steadiness extension & $0.024 \pm 0.011$ & $0.020 \pm 0.008^{*}$ \\
& Force steadiness in flexion & $0.0295 \pm 0.013$ & $0.0279 \pm 0.012$ \\
& RFD & $157 \pm 94$ & $210 \pm 166^{*}$ \\
\cline { 2 - 3 } & & Coefficient $(\boldsymbol{r})$ & -0.3543 \\
\hline Correlation & TTS and extension & $-0.40^{* *}$ & $-0.4063^{*}$ \\
& TTS and flexion & $-0.45^{* *}$ & $-0.443 I^{*}$ \\
& TTS and abduction & $-0.47^{* *}$ & -0.1611 \\
& TTS and RFD & $-0.44^{* *}$ & 0.2040 \\
& TTS and steadiness in extension & $0.34^{* *}$ & 0.6647 \\
\hline
\end{tabular}

Notes: Force: extension, flexion and abduction (measured in $\mathrm{N} \times \mathrm{m}$ ). Force steadiness $\mathrm{CoV}=\mathrm{SD} / \mathrm{mean}$. RFD is measured in $\mathrm{N} \times \mathrm{m} \times \mathrm{s}^{-1}{ }^{*} p<0.05$ and $* * p<0.0 \mathrm{I}$. Abbreviations: TTH, tension-type headache; TTS, total tenderness score; RFD, rate of force development; CoV, coefficient of variation.

total tenderness association with extension and abduction are illustrated in Figure 1A and B, respectively.

\section{Joint mobility}

Only one participant in each group was noted with a positive hypermobility score of $5 / 9$. The mean score in the healthy control group was significantly higher, $0.77 \pm 1.3$, than that in the TTH group, $0.37( \pm 0.95), p=0.036$.

\section{RCT}

In all, 23 patients completed ST and 21 completed EP. In the ST, the mean compliance was attendance at 29.8 out of 30 possible training sessions.

No significant difference was found between the two groups (ST and EP) for any of the test results at the test after 10 weeks or 22 weeks (Table 3 ).

The within-group results for ST showed no significant change in force measurements from baseline to week 10 , but at week 22, a significant increase in abduction $(p=0.033)$ and a significant reduction in both extension $(p=0.031)$ and flexion $(p<0.01)$ were found. Further at week 10, a borderline significant change was found in extension force steadiness ( $p=0.055$ ), and a significant reduction was observed at week $22(p=0.011)$. The RFD had a close to significant increase after 10 weeks, but this was no longer present at week 22 (Figure 2).

The within-group results for EP only showed a significant change in extension force steadiness from baseline to weeks 10 and 22 ( $p=0.001$ and $p<0.01$, respectively).

In summary, the within-group results showed a significant reduction in $\mathrm{CoV}$ for extension force steadiness in both $\mathrm{ST}$ and $\mathrm{EP}$ at week 22. The results indicate a reduced force fluctuation and show that the TTH patients were better at maintaining the force over 25 seconds at week 22 . The intervention results of the test at baseline and weeks 10 and 22 with between-group results and within-group results are presented in Table 3.

\section{Discussion}

This is the first study to examine force steadiness and RFD of the shoulder and to evaluate the effect of ST in the TTH patients in a controlled design.

A lower force steadiness and RFD were shown in the TTH patients compared to the healthy controls. Furthermore, a moderate correlation to tenderness was found. Unfortunately, ST did not have any substantial impact on strength or RFD and did not normalize steadiness or reduce tenderness.

\section{Case-control}

As it was hypothesized, a decreased neuromuscular control in the TTH patients was confirmed by a reduced force steadiness in neck extension and a reduced RFD. The TTH patients had a reduced extension force steadiness with a significant $15 \%$ higher $\mathrm{CoV}$ than the healthy controls. In contrast, no such difference was found in flexion force steadiness. Furthermore, it was confirmed that there was a significant $25 \%$ lower RFD in the TTH group than in the healthy controls. Moderate, although significant, correlations were found between total tenderness and the MVC values in the neck and shoulder muscles, force steadiness and RFD. This suggests that tenderness is associated with the ability to generate force and to control movement in the neck.

\section{RCT}

The reported outcomes are secondary outcomes from the RCT trial. Previously, no significant differences were found in primary outcome between groups, as headache frequency 

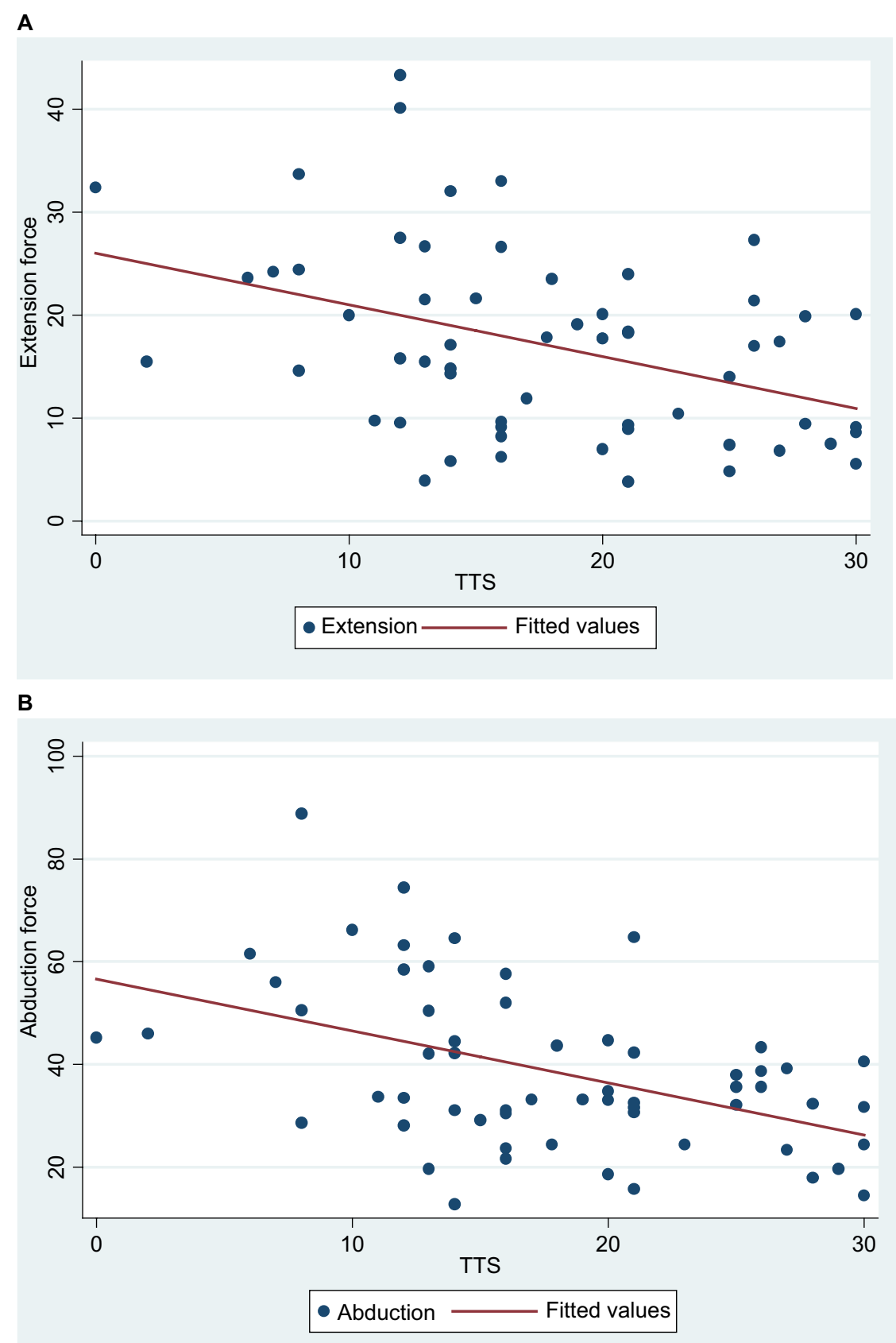

Figure I Scatterplots with fitted regression line representing $60 \mathrm{TTH}$ patients.

Notes: $(A) y$-axis represents extension force $(\mathrm{N} \times \mathrm{m})$, and $\mathrm{x}$-axis represents TTS. (B) $\mathrm{y}$-axis represents abduction force, and $\mathrm{x}$-axis represents TTS.

Abbreviations: TTH, tension-type headache; TTS, total tenderness score.

and duration did not vary between the groups. ${ }^{22}$ In the RCT, we hypothesized that an increase in strength in the ST group would result in a significant improvement in muscular control, shown as a better muscle stability and faster RFD compared to the EP control group.

However, the expected increase in strength supposed to lead to a normalization of the muscle function was not found as there was no significant difference in MVC between groups after the intervention. There is no clear explanation of this outcome as MVC and RFD in other studies have been found to increase with ST. ${ }^{16}$ We found a nonsignificant tendency to an RFD increase from baseline to testing at 10 weeks but a decrease again during the testing at week 22, indicating some response to the 10 weeks of training and a reduction to an RFD even lower than at baseline in the period after training (Figure 2).

As both ST and EP groups improved, the ST intervention did not have a significant effect on force steadiness. 

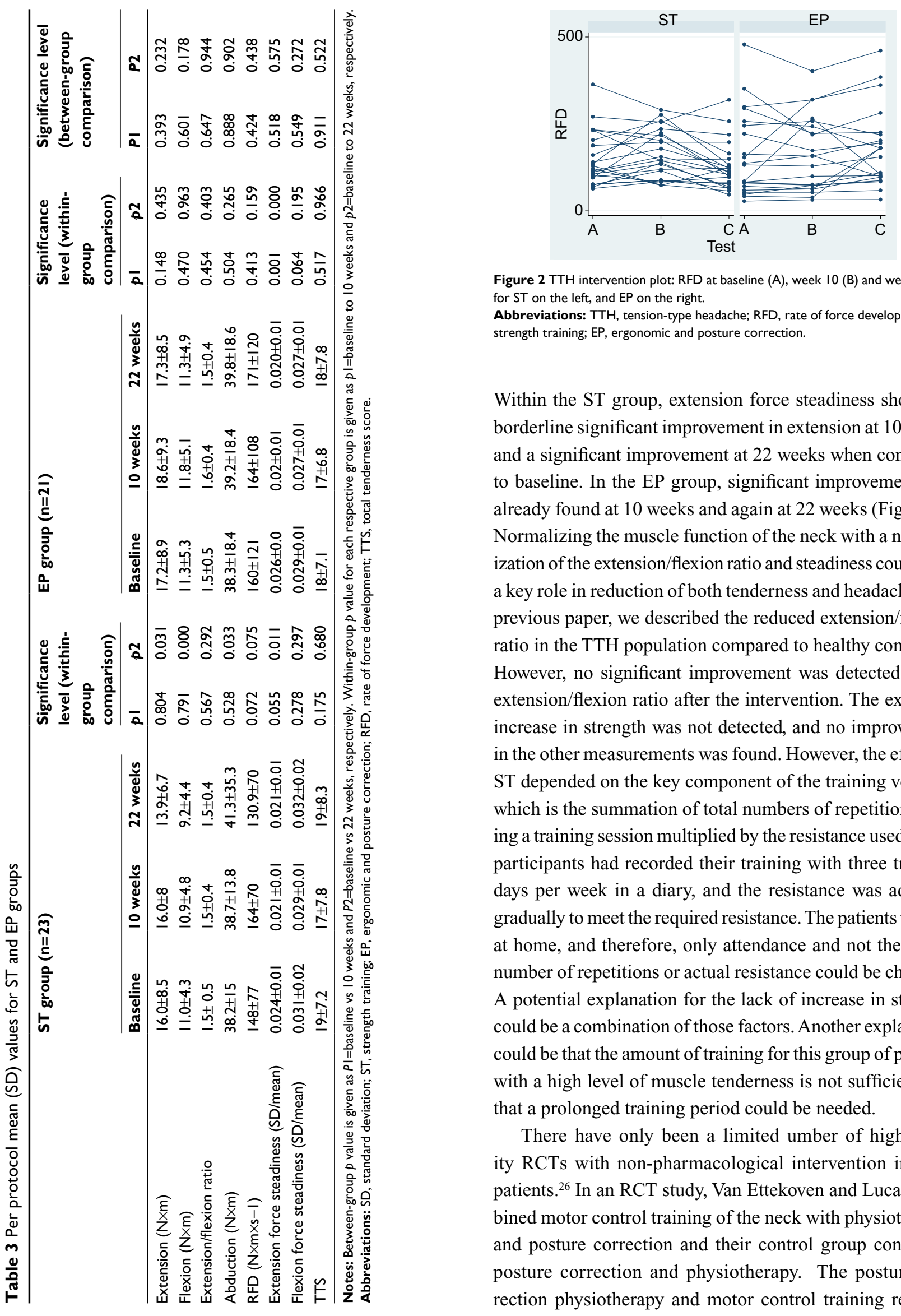

Figure 2 TTH intervention plot: RFD at baseline (A), week 10 (B) and week 22 (C) for ST on the left, and EP on the right.

Abbreviations: TTH, tension-type headache; RFD, rate of force development; ST, strength training; EP, ergonomic and posture correction.

Within the ST group, extension force steadiness showed a borderline significant improvement in extension at 10 weeks and a significant improvement at 22 weeks when compared to baseline. In the EP group, significant improvement was already found at 10 weeks and again at 22 weeks (Figure 2). Normalizing the muscle function of the neck with a normalization of the extension/flexion ratio and steadiness could play a key role in reduction of both tenderness and headache. In a previous paper, we described the reduced extension/flexion ratio in the TTH population compared to healthy controls. ${ }^{13}$ However, no significant improvement was detected in the extension/flexion ratio after the intervention. The expected increase in strength was not detected, and no improvement in the other measurements was found. However, the effect of ST depended on the key component of the training volume, which is the summation of total numbers of repetitions during a training session multiplied by the resistance used. ${ }^{19}$ The participants had recorded their training with three training days per week in a diary, and the resistance was adjusted gradually to meet the required resistance. The patients trained at home, and therefore, only attendance and not the actual number of repetitions or actual resistance could be checked. A potential explanation for the lack of increase in strength could be a combination of those factors. Another explanation could be that the amount of training for this group of patients with a high level of muscle tenderness is not sufficient and that a prolonged training period could be needed.

There have only been a limited umber of high quality RCTs with non-pharmacological intervention in TTH patients. ${ }^{26}$ In an RCT study, Van Ettekoven and Lucas combined motor control training of the neck with physiotherapy and posture correction and their control group conducted posture correction and physiotherapy. The posture correction physiotherapy and motor control training resulted 
in a reduction of headache frequency with 50 percent or more in $82 \%$ after training (6 weeks) and $85 \%$ at follow up (6 months). ${ }^{27}$ No significant differences were found between groups at 6 weeks, but after 6 months, the training group had a significant reduction in frequency, duration and intensity of headaches compared to the control group.

In the present study, we were unable to normalize the muscle function. This could potentially be achieved through training with a combination of interventions. The training could be a combination of training aiming to normalize neck extensors and force steadiness and minimize load on muscles.

\section{Muscle function}

The reduced force steadiness in the TTH group may indicate a dysfunction of the neck muscles, leading to a daily "overuse" of muscle activation to control force. Such an increased activity of the neck muscles could over time lead to tenderness of the neck muscles. ${ }^{18}$ This may be made worse by the reduced RFD in the TTH group. Our previous analysis in the same population as the present study has described a lower extension/flexion ratio at baseline, mainly caused by a lower extension force, and additionally, a borderline significant reduction in abduction force. The combination of a low force steadiness and extension/flexion ratio could be an important key in understanding some of the muscle dysfunction in TTH that may contribute to both muscle tenderness and TTH. With an ongoing increased activity of the neck muscles, a gradual increase in tenderness and TTH may follow, as previously hypothesized by Bendtsen. ${ }^{24}$ Furthermore, the pain-affected muscles could function in a way that is abnormal to their normal motor function in order to divert from pain. Prolonged muscle pain can influence movement patterns. ${ }^{28}$ In the present study, muscle tenderness was associated with the MVC of the neck muscles with a moderate negative correlation that indicates that muscle pain has a negative influence on the force. ${ }^{29} \mathrm{~A}$ potential explanation of the muscle function in the present study is in accordance with the pain model of Lund., et al who has described how muscle pain can modulate muscle activity and lead to an increased activity of antagonists and decreased activity of agonists. ${ }^{30}$ In the present population, the force steadiness and extension/flexion ratio could be explained by an influence of pain on motor control, suggesting that prolonged pain potentially could influence motor pattern. ${ }^{28,29}$

Bendtsen's model for chronification of TTH from 2000 describes how input from painful muscles may contribute to a chronification of the headache. ${ }^{5,24}$ The muscle dysfunction found in the present study may thus potentially present such a muscular impact.
In line with other studies, we expected to and did find a higher degree of tenderness in the TTH group. Furthermore, we found a moderate negative correlation between tenderness and muscle force. This indicates that muscle tenderness is associated with muscle function in the neck and shoulder muscles in TTH patients. ${ }^{7,8}$ Total tenderness has been examined in a 12-year follow-up prospective population-based study, showing that the tenderness was normal at baseline but had increased in the FETTH group..$^{33}$ In the present study, we did not measure the muscle function with electromyography (EMG) which could have contributed further to the understanding of cause and effect of the muscle function in TTH.

In the present study, ST of the shoulders was conducted; this may prove not to be sufficient in reducing highly frequent TTH. It could be necessary to address the neck muscles more directly through training to normalize the neck function.

The decrease in muscle tenderness following ST could therefore play a key role in reducing TTH.

\section{Strengths and limitations}

A strength of the present study was the precise diagnosis of the headache patients. Furthermore, the systematic registration of the tenderness made it possible to contribute further to the understanding of how muscles work in TTH patients. A limitation of the study was that neck pain was not prospectively registered; hence, it is unknown if the training activity decreased neck pain. An unexpected finding was that the ST group did not have increased muscle strength as expected. Because ST is - per definition - training that increases muscle strength, the participants of the present study are unlikely to have self-trained sufficiently, e.g., the intensity of training at home may have been too low. As the training frequency and length of the intervention are quite similar to other studies that have shown clear increases in muscle strength, it is likely that too low training intensity and/or the severity of headache with a high level of tenderness were the major causes of lack of progression. Potentially, longer training periods and higher intensity ST could have contributed to a further impact on functions of muscle. Lack of control of training doses in each session, due to home training, could potentially have influenced the results. The power calculation was done regarding the primary outcome; therefore, lack of power could potentially explain some of the results.

\section{Conclusion}

A lower force steadiness and RFD were shown in the TTH patients compared to the healthy controls. Furthermore, a moderate correlation to tenderness was found. Unfortunately, 
ST did not have any substantial impact on strength or RFD and did not normalize steadiness or reduce tenderness. An improved intervention that could reduce tenderness should be examined in a future study. That study could include both posture correction and more intensive and prolonged training to normalize neck extensors and force steadiness in the RCT study design.

\section{Key points}

- TTH patients compared to the healthy controls showed a deteriorated muscle function, indicated by a lower force steadiness and RFD.

- A significant $(p<0.01)$ and moderate correlation to muscle tenderness was found.

- The effect of ST was not larger than EP as both groups of TTH patients showed some improvement in neck and shoulder function during the 10 weeks intervention.

\section{Acknowledgment}

This study was supported by the Tryg Foundation.

\section{Disclosure}

The authors report no conflicts of interest in this work.

\section{References}

1. Lyngberg AC, Rasmussen BK, Jørgensen T, Jensen R. Has the prevalence of migrane and tension type headache changed over a 12 year period. Eur J Epidemiol. 2005;20(3):243-249.

2. Rasmussen BK, Jensen R, Schroll M, Olesen J. Epidimiologi of headache in a general population. A prevalence study. J Clin Epidimiol. 1991;44(11):1147-1157.

3. Schwartz BS, Stewart WF, Lipton RB. Lost workdays and decreased work effectiveness associated with headache in the workplace. J Occup Environ Med. 1997;39(4):320-327.

4. Steiner TJ, Stovner LJ, Katsarava Z. The impact of headache in Europe: principal results of the Eurolight. J Headache Pain. 2014;15:31.

5. Bendtsen L, Fernández-de-la-Peñas C. The role of muscles in tensiontype headache. Curr Pain Headache Rep. 2011;15(6):451-458.

6. Jensen R. Peripheral and central mechanisms in tensiontype headache: an update. Cephalalgia. 2003;23(suppl 1):49-52.

7. Jensen R. Pathophysiological mechanisms of tension-type headache: a review of epidemiological and experimental studies. Cephalalgia. 1999;19(6):602-621.

8. Langemark M, Olesen J. Pericranial tenderness in tension headache. Cephalalgia. 1987;7:249-255.

9. Hagen K, Einarsen C, Zwart J, Svebak S, Bovim G. The co-occurrence of headache and musculoskeletal symptoms amongst 51050 adults in Norway. Eur J Neurol. 2002;9(5):527-533.

10. Ashina S, Bendtsen L, Lyngberg AC, Lipton RB, Hajiyeva N, Jensen R. Prevalence of neck pain in migraine and tension-type headache: a population study. Cephalalgia. 2015;35:211-219.

11. Muceli S, Farina D, Kirkesola G, Katch F, Falla D. Reduced force steadiness in women with neck pain and the effect of short term vibration. J Electromyogr Kinesiol. 2011;21(2):283-290.

12. Fernández-de-las-Peñas C, Falla D, Arendt-Nielsen L, Farina D. Cervical muscle co-activation in isometric contractions is enhanced in chronic tension-type headache patients. Cephalalgia. 2008;28(7):744-751.
13. Madsen BK, Søgaard K, Andersen LL, Skotte JH, Jensen RH. Neck and shoulder muscle strength in patients with tension-type headache: a case-control study. Cephalalgia. 2016;36(1):29-36.

14. Andersen LL, Holterman A, Jørgensen MB, Sjøgaard G. Rapid muscle activation and force capacity in conditions of chronic muscleskeletal pain. Clin Biomech. 2008;23:1237-1242.

15. Andersen LL, Nielsen PK, Søgaard K, Andersen CH, Skotte J, Sjøgaard G. Torque-EMG-velocity relationship in female workers with chronic neck muscle pain. J Biomech. 2008;41(9):2029-2035.

16. Andersen LL, Kjaer M, Sogaard K, Hansen L, Kryger AI, Sjogaard G. Effect of two contrasting types of physical exercise on chronic neck muscle pain. Arthritis Rheum. 2008;59(1):84-91.

17. Biolo G, Maggi SP, Williams BD, Tipton KD, Wolfe RR. Increased rates of muscle protein turnover and amino acid transport after resistance exercise in humans. Am J Physiol. 1995;268(3 pt 1):E514-E520.

18. Sjogaard G, Lundberg U, Kadefors R. The role of muscle activity and mental load in the development of pain and degenerative processes at the muscle cell level during computer work. Eur J Appl Physiol. 2000;83(2-3):99-105.

19. Kraemer WJ, Adams K, Cafarelli E, et al. American College of Sports Medicine position stand. Progression models in resistance training for healthy adults. Med Sci Sports Exerc. 2002;34(2):364-380.

20. Headache Classification Subcommittee of the International Headache Society. The international classification of headache disorders. 2nd ed. Cephalalgia. 2004;24(suppl 1):1-159.

21. Jull G, Amiri M, Bullock-Saxton J, Darnell R, Landeret C. Cervical musculoskeletal impairment in frequent intermittent headache part 1: subjects with single headaches. Cephalalgia. 2007;27:793-802.

22. Madsen BK, Søgaard K, Andersen LL, Tornøe B, Jensen RH. Efficacy of strength training on tension-type headache. A randomized controlled study. Cephalalgia. Epub 2017 Jan 1.

23. Gram B, Andersen C, Zebis MK, et al. Effect of training supervision on effectiveness of strength training for reducing neck/shoulder pain and headache in office workers: cluster randomized controlled trial. Biomed Res Int. 2014;2014:693013.

24. Bendtsen L. Central sensitization in tension-type headache possible pathophysiological mechanisms. Cephalalgia. 2000;20(5):486-508.

25. Juul B, Rogind H, Jensen DV, Remvig L. Inter-examiner reproducibility of tests and criteria for generalized joint hypermobility and benign joint hypermobility syndrome. Rheumatology. 2007;46(12):1835-1841.

26. Luedtke K, Allers A, Schulte LH, May A. Efficacy of interventions used by physiotherapists for patients with headache and migraine-systematic review and meta-analysis. Cephalalgia. 2016;36(5):474-492.

27. Van Ettekoven H, Lucas C. Efficacy of physiotherapy including a craniocervical training programme for tension- type headache; a randomized clinical trial. Cephalalgia. 2006;26(8):983-991.

28. Hodges PW, Tucker K. Moving differently in pain: a new theory to explain the adaptation to pain. Pain. 2011;152(3 suppl):90-98.

29. Graven-Nielsen T, Svensson P, Arendt-Nielsen L. Effects of experimental muscle pain on muscle activity and coordination during static and dynamic motor function. Electroencephalogr Clin Neurophysiol. 1997;105(2):156-164.

30. Lund JP, Donga R, Widmer CG, Stohler CS. The pain-adaptation model: a discussion of the relationship between chronic musculoskeletal pain and motor activity. Can J Physiol Pharmacol. 1991;69(5):683-694.

31. Aaseth K, Grande RB, Lundqvist C, Russell MB. Pericranial tenderness in chronic tension-type headache: the Akershus population-based study of chronic headache. J Headache Pain. 2014;15:58.

32. Andersen LL, Mortensen OS, Zebis MK, Jensen RH, Poulsen OM. Effect of brief daily exercise on headache among adults - secondary analysis of a randomized controlled trial. Scand JWork Environ Health. 2011;37(6):547-550.

33. Buchgreitz L, Lyngberg AC, Bendtsen L, Jensen R. Increased pain sensitivity is not a risk factor but a consequence of frequent headache: a population-based follow-up study. Pain. 2008;137(3):623-630. 
The Journal of Pain Research is an international, peer reviewed, open access, online journal that welcomes laboratory and clinical findings in the fields of pain research and the prevention and management of pain. Original research, reviews, symposium reports, hypothesis formation and commentaries are all considered for publication.

Submit your manuscript here: https://www.dovepress.com/journal-of-pain-research-journal

The manuscript management system is completely online and includes a very quick and fair peer-review system, which is all easy to use. Visit http://www.dovepress.com/testimonials.php to read real quotes from published authors. 